\title{
Research Article \\ Scattering of Electric Field from Leaky Coaxial Cable in Confined Area
}

\author{
Asad Saleem, Min Wang, and Guoxin Zheng \\ Shanghai Institute for Advanced Communication and Data Science (SICS), Shanghai University, Shanghai, China \\ Correspondence should be addressed to Guoxin Zheng; gxzheng@staff.shu.edu.cn
}

Received 26 April 2017; Revised 22 June 2017; Accepted 25 July 2017; Published 16 October 2017

Academic Editor: Ikmo Park

Copyright (C) 2017 Asad Saleem et al. This is an open access article distributed under the Creative Commons Attribution License, which permits unrestricted use, distribution, and reproduction in any medium, provided the original work is properly cited.

The new radiation model for the electric field scattering of single leaky coaxial cable (LCX) being a transmitting antenna in the rectangular blind zone at $1.8 \mathrm{GHz}$ is reported. The effect of varying the receiving antenna distance from the LCX on the fluctuation of radiated field using Ray-Tracing Method is examined. The line of sight and reflected paths between the LCX and receiving antenna for both horizontal and vertical polarizations are considered. Furthermore, it is inferred that the selection of appropriate period of slots and distance of the receiver reduce the fluctuations of electric field. The theory and procedures used in this paper can be extrapolated in different slot patterns of the LCX and zones which have many valuable applications for mobile communication in underground mines, tunnels, and railways.

\section{Introduction}

Leaky coaxial cables have found many useful applications in communication systems both as feeders and as transmitting antennas such as Communication Based Train Control (CBTC) systems, for high speed railway, automated highway, wireless LAN retrieving, location of train, and underground conveyance [1]. Mobile edge service platform delivers dynamical sensor networks (SNS) in the high speed rail (HSR). Passengers acquire information about train location and environment by interacting with a virtual crew through dynamical SNS application via their smartphones [2]. The Long Term Evolution for Metro (LTE-M) method using the LCX for the CBTC system has been found to be an important communication technique for upcoming urban rail transit system. The LTE-M is designed for high speed wireless communications in confined areas. The key requirements for the LTE-M are high peak data rates, high spectral efficiency, less processing time, and flexibility in bandwidth [3]. Experimental visualization of the LCX MIMO system in tunnel environment is demonstrated in [4] and it is inferred that the LCX MIMO system performance is not fully dependent on the LCX spacing. The Finite-Difference Time-Domain (FDTD) is a method to visualize the whole wireless radio frequency (RF) connection of the LCX in a tunnel environment. The FDTD method is used to predict the radiation patterns of cylindrical coordinates and it covers a wide range of frequencies with a single simulation run [5].

LCX is modeled in different shapes according to requirements. In order to avoid transmission loss inside tunnel environment, Fujikura has developed a lightweight and thin LCX-5D that is capable of securing a stable communication region near the LCX [6]. In [7], patched leaky coaxial cable (PLCX) is considered as a substitute for the conventional LCX for a wireless connection in a composite environment to acquire smoother radiated field coverage. Leaky cable has slots on its outer conductor, which allows radio signals to pass in and out of its surface along the whole length of the cable [8]. Radio communication in the rectangular blind zone depends on the magnitude of the electromagnetic field intensity (EMI), environmental conditions nearby the leaky cable, and installation methods [9]. By increasing the number of slots of the LCX, it brings down the resonance centerfrequency and provides broad radiation bandwidth and good radiation efficiency [10].

The influence of slot period on the performance of the LCX MIMO system in terms of capacity, correlation, and channel matrix rank is discussed in [11], when single LCX is used as two transmitting antennas. Ray-Tracing (RT) method is used for precise prediction of radio channel constraints and 


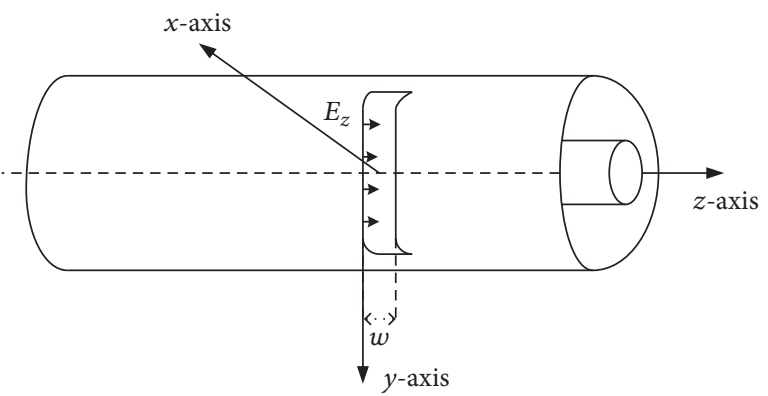

(a)

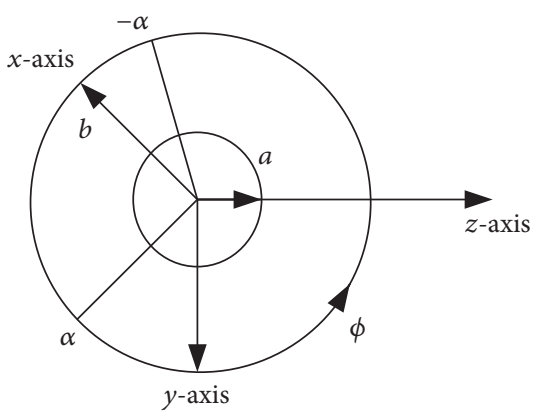

(b)

FIGURE 1: The slot structure of LCX.

significant accuracy of near-field computations for indoor environment. In [12], the authors describe the importance of the RT propagation modeling for the forthcoming smallcell millimeter-wave schemes and emphasize the significance of the application potential. In [13], two major kinds of LCXs, coupled mode and radiating mode, are explained concisely for an indoor building environment. The Akaike information criterion (AIC) can be used to find out the model of small-scale fading. It is revealed that lognormal distribution is appropriate for small-scale fading, and the representing parameters of lognormal distribution are also found from the experimental results. The field distribution in the tunnel area is discussed under the excitations of the LCX and BSA. To include the Tx/Rx nearby environment, an IMT including the UPML technique and the FDTD method is applied [14]. In [15], the authors explained the tunnel antenna which develops circularly polarized waves alongside its axis and has an improved directivity. Though the literature conveys several studies, many of these works lack experimental validations of real time dimensions of tunnel and practical antenna location.

The objective of this study is to propose a radiation model of the LCX, which explore the insights of the LTE used in China's metro at $1.8 \mathrm{GHz}$. Radiation from a leaky coaxial cable in a tunnel environment is studied for both line of sight (LOS) path and reflected paths (NLOS) based on RT method. We studied the effect of varying the period of slots on radiated field and compared theoretically the design in an actual environment for both horizontally and vertically polarized radio waves. The measurement results may be useful to identify the shortcomings in LTE systems and to determine an optimum system design based on the LCX deployment in a real time tunnel environment.

\section{Theory of Radiations}

Theory of radiation deals with transmission or emission of energy in the form of waves from a medium such as electromagnetic radiation. The intensity of these radiations follows the inverse square law of distance from their origin. Generally, these radiations depend on two factors: (1) scattering of magnetic current of a slot and (2) radiated field of a slot. The following section will explain these two problems briefly.
2.1. Scattering of Magnetic Current of Slot. The procedure of calculating the scattered magnetic current of a slot is complex. In this paper, we deal only with the vertical and horizontal slotted leaky coaxial cable as shown in Figures 1(a) and 1(b). The LCX slots are vertical in shape and they are perpendicular to $z$-axis. Here, we considered the slot angle as $2 a$, where $L$ represents length of cable, $l$ is the length of slot, $a$ and $b$ represent the inner and outer radius of the conductor, and $w$ denotes the width of the slot. The magnetic current of the cable is described in the following equations [9].

$$
M=C\left[1-\frac{\cos \left(K_{0} b \varphi\right)}{\cos \left(k_{o} b \alpha\right)}\right],
$$

where

$$
\begin{aligned}
& C=\frac{\left(-j V_{0} \sqrt{\varepsilon_{r}}\right)}{\left[2 \varphi k_{0} b \ln (b / a)\right]} \\
& \varphi=\int_{-b \alpha}^{b \alpha}\left(\frac{e^{-j K_{0} R}}{2 \pi R}\right) d l, \\
& R=\sqrt{l^{2}+\left(\frac{w}{2}\right)^{2}} .
\end{aligned}
$$

$\varepsilon_{r}$ is the dielectric coefficient of cable, $k_{0}$ is the wave number of the free space, and $V_{0}$ is excited voltage of LCX.

2.2. Radiated Field of Slot. Field produced along leaky cable due to static and moving charges is considered as an electric and magnetic field, respectively. The LCXs have two types of emitting modes which are surface mode and radiating mode. The waves exist around the LCX in emitting mode and the waves radiate in far field only in radiating mode [16]. On the basis of cylindrical Fourier transforms of $E_{z}$ and $E_{\varphi}$, we can find the distribution of the electric field due to the slot on the outer conductor of LCX [9] as shown in Figure 2.

$$
E_{z}(\varphi, z)=\frac{V}{w}\left(1-\frac{\cos \left(k_{0} b \varphi\right)}{\cos \left(k_{0} b \alpha\right)}\right),
$$

where $V=\left(-j V_{0} \sqrt{\varepsilon_{r}}\right) /\left[2 k_{0} b \ln (b / a)\right], E_{z}$ shows the electric field along $z$-axis, and $o$ represents the location of slot. 


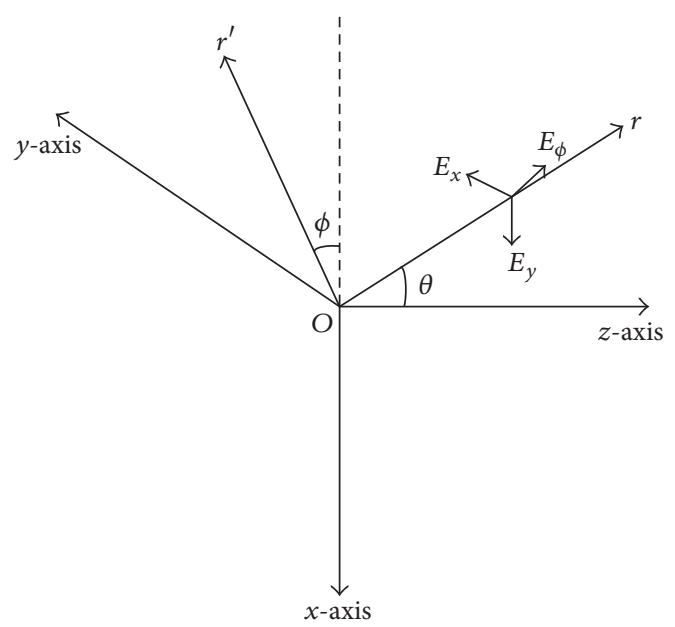

FIGURE 2: Radiated field of the LCX.

Distance from point $o$ to one point in space is $r$ whose projection in xoy plane is $r^{\prime} . \theta$ is the angle between $r$ and $z$ axis while $\varphi$ is the angle between $r^{\prime}$ and $x$-axis.

$$
\begin{aligned}
E_{\varphi} & =-j k_{0} \frac{e^{-J k_{0} r}}{\pi r \epsilon_{0}} \sin \theta \sum_{n=-\infty}^{\infty} e^{j n \varphi} j^{n+1} g_{n}\left(-k_{0} \cos \theta\right), \\
g_{n}(\omega) & =\frac{\epsilon_{0} n \omega \widetilde{E_{z}}(n, \omega)}{b\left(K_{0}{ }^{2}-\omega^{2}\right)^{3 / 2} H_{n}{ }^{2}\left(b \sqrt{k_{0}{ }^{2}-\omega^{2}}\right)},
\end{aligned}
$$

where $\omega$ is the angular frequency, $\epsilon_{0}$ is dielectric coefficient of free space, and $H_{n}{ }^{2}$ is Hankel function of 2nd type of rank $n_{\mathrm{th}} \cdot \widetilde{E_{z}}$ is cylindrical Fourier transform of $E_{z}$.

$$
\widetilde{E_{z}}(n, \omega)=\frac{1}{2 \pi} \int_{0}^{2 \pi} d \varphi \int_{-\infty}^{\infty} d z E_{z}(\varphi, z) e^{-j n \varphi} e^{-j \omega z} .
$$

From the above formulation, we can write it as

$$
E_{\varphi}=V(\theta, \varphi) \frac{e^{-j k_{0} r}}{r} \sin \theta
$$

where

$$
\begin{aligned}
& V(\theta, \varphi)=\frac{j V \cos \theta}{\pi^{2} b k_{0} \sin ^{3} \theta} \sum_{n=-5}^{n=+5} \frac{n e^{j n \varphi} j^{n+1}}{H_{n}^{(2)^{\prime}}\left(b k_{0} \sin \theta\right)} \\
& \cdot\left(\frac{\sin n \alpha}{n}-\frac{m \sin m \alpha \cos n \alpha-n \cos m \alpha \sin n \alpha}{\left(m^{2}-n^{2}\right) \cos m \alpha}\right),
\end{aligned}
$$

where $m=k_{0} b . E_{z}$ remains invariable along $z$-axis because width $w$ of slot is very small. The scattered field due to circumferential polarization can be calculated from (6) and (7).

2.3. The Pathway of Electric Waves in Rectangular Blind Zone. We placed the LCX along $z$-axis and the receiver $(\mathrm{Rx})$ is placed at a far distance from the LCX. The pathway of electric waves is shown in three-dimensional spaces in
Figures 3(a), 3(b), and 3(c). Distance between the LCX and Rx is considered along $x$-axis and LCX is placed at $1.1 \mathrm{~m}$ higher than the receiving antenna along $y$-axis as shown in Figure 4. The vertically polarized components of $E_{\varphi}$ can be written by using Ray-Tracing Method [17-19].

$$
E_{y}=V\left(\theta_{i}, \varphi_{i}\right) \frac{e^{-j k_{0}\left(r_{i}+r_{i 1}\right)-j \beta_{i} P}}{r_{i}+r_{i 1}} \sin \theta_{i} \cos \varphi_{i} \Gamma_{h},
$$

where

$$
\begin{aligned}
& r_{i}=\sqrt{x_{o}^{2}+y_{0}^{2}+z_{0}^{2},} \\
& r_{i 1}=\sqrt{x_{0}^{2}+\left(h-h_{0}\right)^{2}+\left(i p-z_{0}\right)^{2}}, \\
& \varphi_{i}=\arccos \left(\frac{x_{0}}{\sqrt{x_{0}^{2}+h_{0}^{2}}}\right), \\
& \theta_{i}=\arctan \left(\frac{\sqrt{x_{0}^{2}+h_{0}^{2}}}{z_{0}}\right), \\
& \beta=k_{0} \sqrt{\epsilon_{r}}, \\
& \gamma=\arctan \left(\frac{x_{0}}{\sqrt{z_{0}^{2}+h_{0}^{2}}}\right) .
\end{aligned}
$$

Height of receiving antenna is $h=1.6 \mathrm{~m}$, while the height of the LCX is $h_{0}=2.7 \mathrm{~m} . \Gamma_{h}$ is a reflection coefficient of horizontally polarized waves, $\left(\epsilon_{1}-j \epsilon_{1}^{\prime}\right)$ is a complex dielectric coefficient of the wall, and $z_{0}$ is the remaining distance starting from current slot to the remaining $z$-axis. The reflection coefficient for the vertically polarized waves is given below.

$$
\Gamma_{v}=\frac{\left(\epsilon_{1}-j \epsilon_{1}^{\prime}\right) \sin \gamma-\sqrt{\left(\epsilon_{1}-j \epsilon_{1}^{\prime}\right)-\cos ^{2} \gamma}}{\left(\epsilon_{1}-j \epsilon_{1}^{\prime}\right) \sin \gamma+\sqrt{\left(\epsilon_{1}-j \epsilon_{1}^{\prime}\right)-\cos ^{2} \gamma}} .
$$

The reflection coefficient of a horizontally polarized wave is written as

$$
\Gamma_{h}=\frac{\sin \gamma-\sqrt{\left(\epsilon_{1}-j \epsilon_{1}^{\prime}\right)-\cos ^{2} \gamma}}{\sin \gamma+\sqrt{\left(\epsilon_{1}-j \epsilon_{1}^{\prime}\right)-\cos ^{2} \gamma}} .
$$

The total radiated field is calculated by adding the effect of all slots of the leaky coaxial cable:

$$
E_{y \Sigma}=\sum_{i=1}^{N} V\left(\theta_{i}, \varphi_{i}\right) \frac{e^{-j k_{0}\left(r_{i}+r_{i 1}\right)-j \beta_{i} P}}{r_{i}+r_{i 1}} \sin \theta_{i} \cos \varphi_{i} \Gamma_{v} .
$$

For reflected waves, this method is also applicable. By using superposition principle, we obtain the total radiated field produced by the LCX at the receiving antenna. 


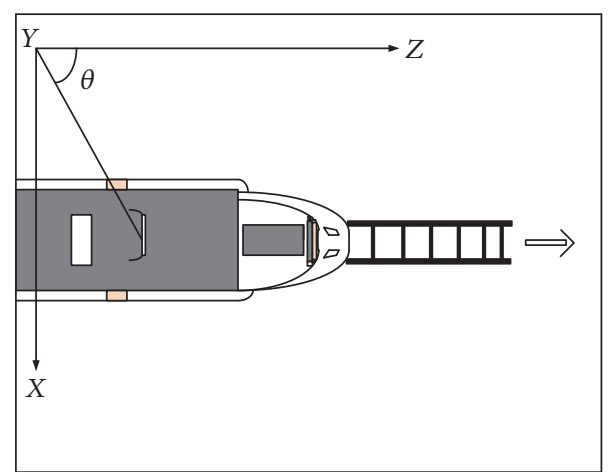

(a)

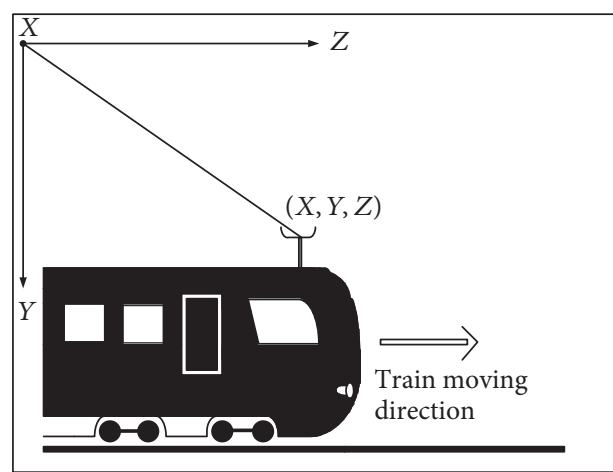

(b)

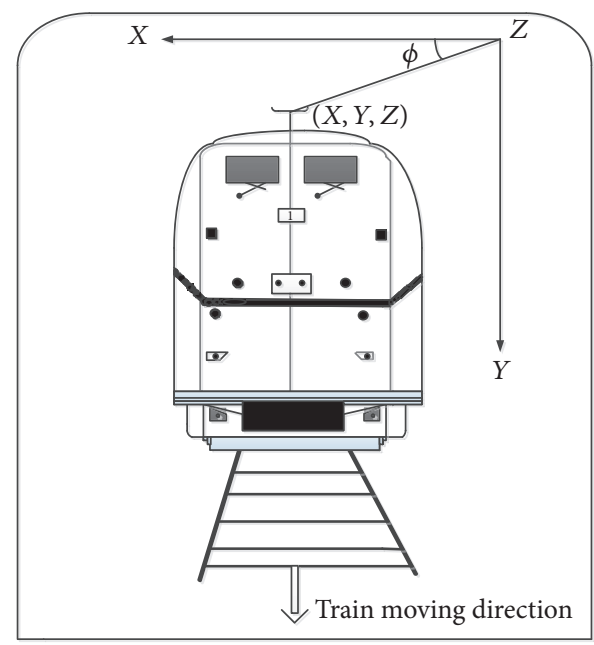

(c)

Figure 3: The three-dimensional view of radiations: (a) the nether view, (b) the side view, and (c) the obverse view.

\section{Experimental Description and Numerical Results}

The measurements were carried out in the subway-like tunnel of Zhongtian RF Cable Co., Ltd., at Nantong, China. The length of the tunnel was $100 \mathrm{~m}$, with the cross-section of the first $50 \mathrm{~m}$ arch and the following $50 \mathrm{~m}$ rectangle, but we considered the first $50 \mathrm{~m}$ arch area for measurements. The wall of the tunnel was covered with concrete material. Nobody was moving throughout the experiments. The parameters of the measurement setup are given in Table 1 . In these calculations, the radius of outer conductor of LCX was $16 \mathrm{~mm}$ and radius of inner conductor was $6 \mathrm{~mm}$. The volume of the rectangular blind zone was $50 \mathrm{~m}$ (length) $\times 5 \mathrm{~m}$ (width) $\times 3 \mathrm{~m}$ (height) The length of the cable was $50 \mathrm{~m}$ along the $z$-axis and the minimum distance between the Rx point and the LCX along $x$-axis was $2 \mathrm{~m}$.

We divided the measurement area into three different regions as Region 1, Region 2, and Region 3. Each region has 15 observing points. We further divided these points into $3 \times 5$ matrix formats; the distance between each observing point was $0.5 \mathrm{~m}$ as illustrated in Figure 4. The LCX was $1.1 \mathrm{~m}$ higher than a conventional receiving antenna. Height of LCX was $2.7 \mathrm{~m}$ and the slot angle was taken as $\pi / 4$. The complex
TABLE 1: Measuring parameters.

\begin{tabular}{lc}
\hline Unit & Parameter \\
\hline Slots distance of LCX & $0.6 \mathrm{~m}$ \\
Transmitted power & $23 \mathrm{dBm}$ \\
Bandwidth & $40.8 \mathrm{MHz}$ \\
Tx-antenna & ZTT-LCX of $50 \mathrm{~m}$ length \\
Rx-antenna & UHA9125D dipole antenna \\
Antenna gain & $2.15 \mathrm{dBi}$ \\
Height of Tx-antenna & $2.7 \mathrm{~m}$ \\
Height of Rx-antenna & $1.6 \mathrm{~m}$ \\
Sampling rate & $81.6 \mathrm{MHz}$ \\
Resistance & $50 \mathrm{ohm}$ \\
Measurement time & $50 \mathrm{~ms}$ \\
\hline
\end{tabular}

dielectric coefficient was $4-j 0.3$ and the dielectric coefficient of dielectric sheet was 1.25 . The frequency was considered $1.8 \mathrm{GHz}$ because this frequency spectrum provides broader LTE coverage as well as improved indoor signal strength, whereas the excitation voltage was considered as unity.

The required number of slots can be determined using the relation $N=L / P-1$. Period of slots of the LCX have great 

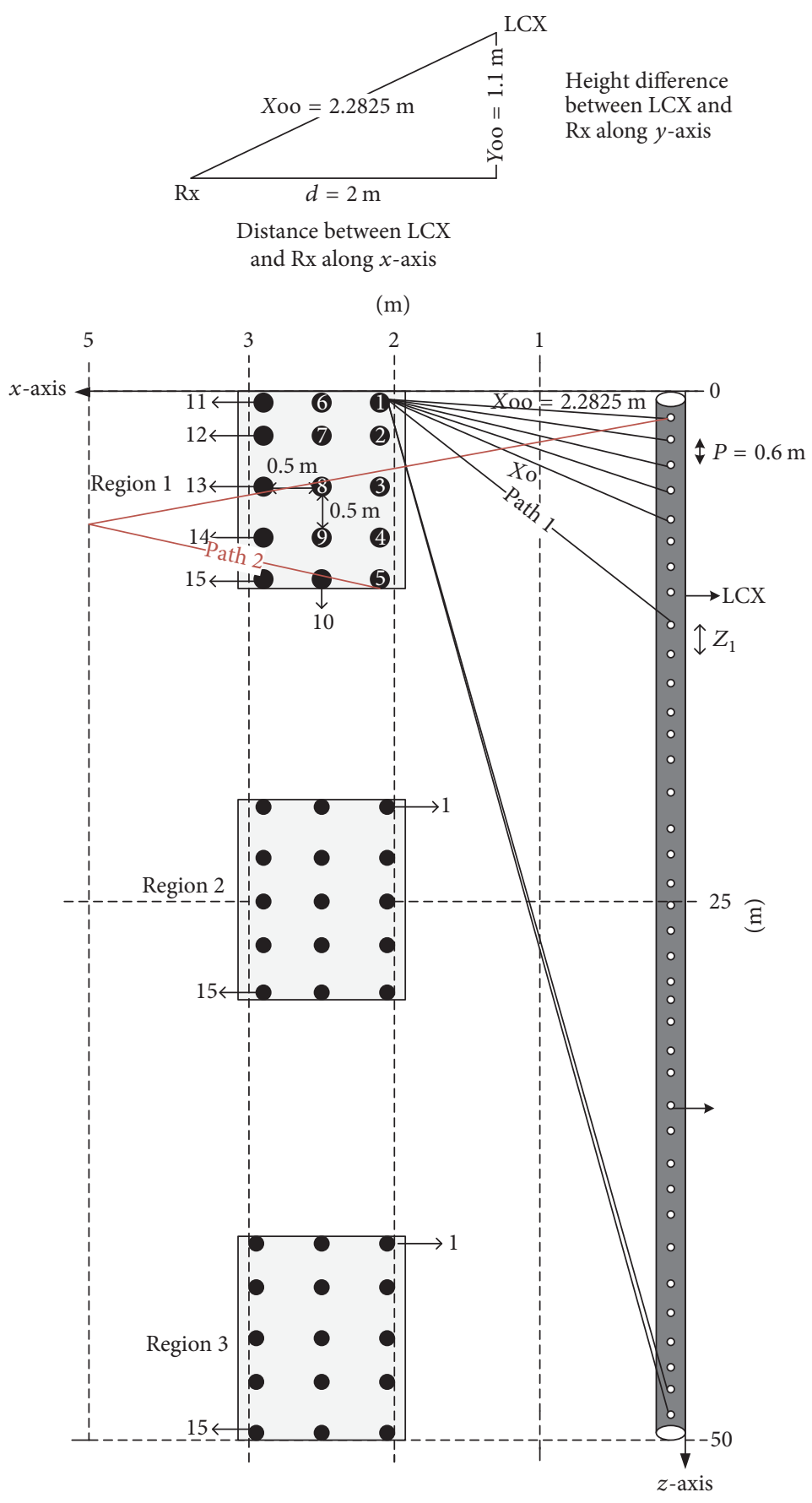

Path 1: LOS path between LCX and receiver $i$ : number of slots Height of LCX: $2.7 \mathrm{~m}$ Height of Rx: 1.6 m

- Measurement point of Rx antenna Path 2: reflected path

FIgURE 4: The visualization of Nantong Tunnel area.

impact on the radiated field. Desired results can be obtained by adjusting the proper period of slots. Minimum slots period of the LCX provide better radiated field such that $P=0.4 \mathrm{~m}$ shows improved performance more than others, as specified in Figure 5.

By using MATLAB Software, we analyzed the effect of radiated field $E_{y}$ due to fastened LCX and compared it with different positions along all three regions of subway-like tunnel.

3.1. Horizontal Polarization. In this experiment, we considered the horizontally polarized radio waves radiated from the leaky coaxial cable along the $z$-axis and evaluated its effect on the receiving antenna which was horizontally polarized 


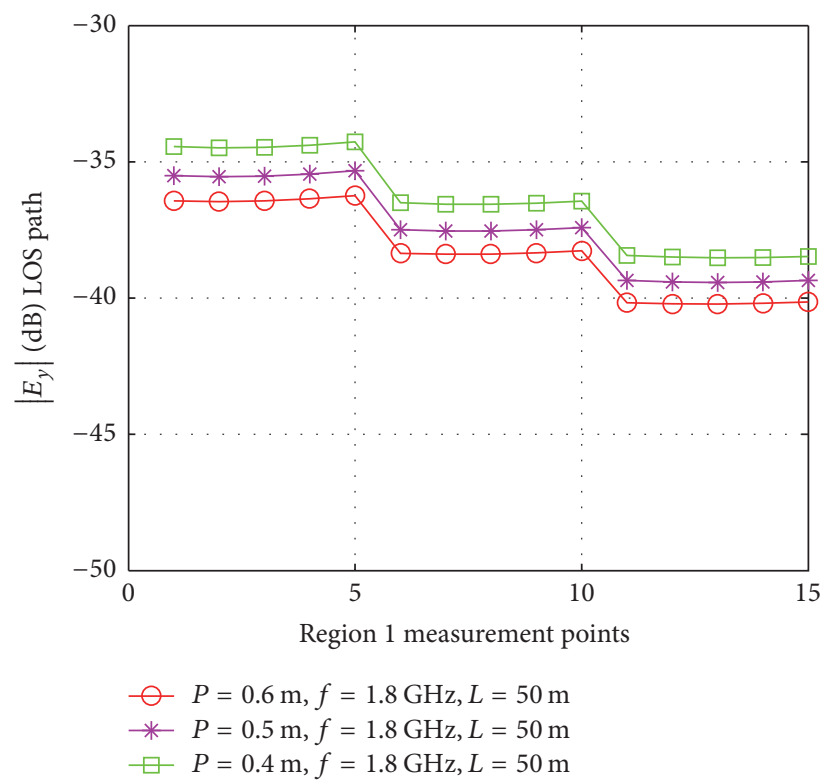

FIgURE 5: The distribution of $E_{y}$ for different period of slots of horizontally polarized LCX in Region 1 for LOS path.

and it was located at minimum distance of $2 \mathrm{~m}$ away from the LCX along the $x$-axis. We extracted the LOS path from the total radiated path using the Ricean $K$-factor method [20]. This estimated moment method provides a satisfactory resemblance with the actual experimental data. To save space, mathematical formulas for the moment method are not covered here. Further details can be determined in [20]. We only give final results here; that is,

$$
K=\frac{|V|^{2}}{\sigma^{2}}
$$

where

$$
\begin{aligned}
|V|^{2} & =\left[G_{a}{ }^{2}-G_{v}{ }^{2}\right]^{1 / 2}, \\
\sigma^{2} & =G_{a}-\left[G_{a}{ }^{2}-G_{v}{ }^{2}\right]^{1 / 2} .
\end{aligned}
$$

Here $|V|^{2}$ and $\sigma^{2}$ give LOS and NLOS received powers, respectively. Further, $G_{a}$ represents the first moment of received power gain and $G_{v}$ is the real value of the second moment which is the root mean square ( $\mathrm{rms}$ ) variation of the corresponding power gain around $G_{a}$. First path contains only the LOS path which is illustrated as Path 1, while the second part (Path 2) consists of the NLOS paths containing reflection from roof, wall, and floor as illustrated in Figure 4. Transmitted power and resistance $(R)$ along LCX were considered, respectively, as $23 \mathrm{dBm}$ and $50 \mathrm{ohm}$. The height of the LCX was $2.7 \mathrm{~m}$ from floor and the height of receiving antenna was $1.6 \mathrm{~m}$. We divided the concern area into three different regions of same length. We further compared these theoretical results with the experimental data which was conducted in the Nantong subway-like tunnel. First comparison consists of the LOS path components extracted from the real time data evaluation of the Nantong tunnel and simulated results (MATLAB results based on theory), while second comparison consists of both the LOS and NLOS components of simulations and Nantong tunnel experimental data as illustrated in Figure 6(a). Results show good agreement between the simulated and real time calculated data. From Figure 6(a), it is evident that in Region 1 when period of slots is $0.6 \mathrm{~m}$ and length of cable is $50 \mathrm{~m}$ then $E_{y}$ variations of vertically slotted LCX are stable. Same procedure was repeated for Region 2 and Region 3 as given in Figures 6(b) and 6(c), respectively, and the results show similarity with the former experiments of Region 1.

3.2. Vertical Polarization. In order to further investigate the effect of radiated electric field, we considered the vertically polarized LCX of $0.6 \mathrm{~m}$ of period of slots and $50 \mathrm{~m}$ along $z$ axis, locating a receiving antenna of vertically polarized radio waves at $2 \mathrm{~m}$ distance from the LCX along $x$-axis following Path 1 and Path 2 as clarified in previous experiment. Then the overall radiated field can be achieved by accumulating the effect of all the slots on LCX using (12). The theory and measurement data are compared for both the LOS and NLOS paths and it proves resemblance in behavior as given in Figure 7. In Region 1, Region 2, and Region 3, $E_{y}$ shows variations as the distance of reference points of respective regions increase along $z$-axis as specified in Figures 7(a), 7(b), and $7(\mathrm{c})$.

From Figures 6 and 7, it is evident that the period of slots, length of cable, and the separation between antennas have a significant impact on the magnitude of radiated field. By proper selection of slots period, stable $E_{y}$ can be achieved which is essential for radio communication. It is also clear that, in Region 2, we have a strong radiated field as compared to Region 1 and Region 3 in both horizontally polarized and vertically polarized cases, whereas Region 1 has lowest 


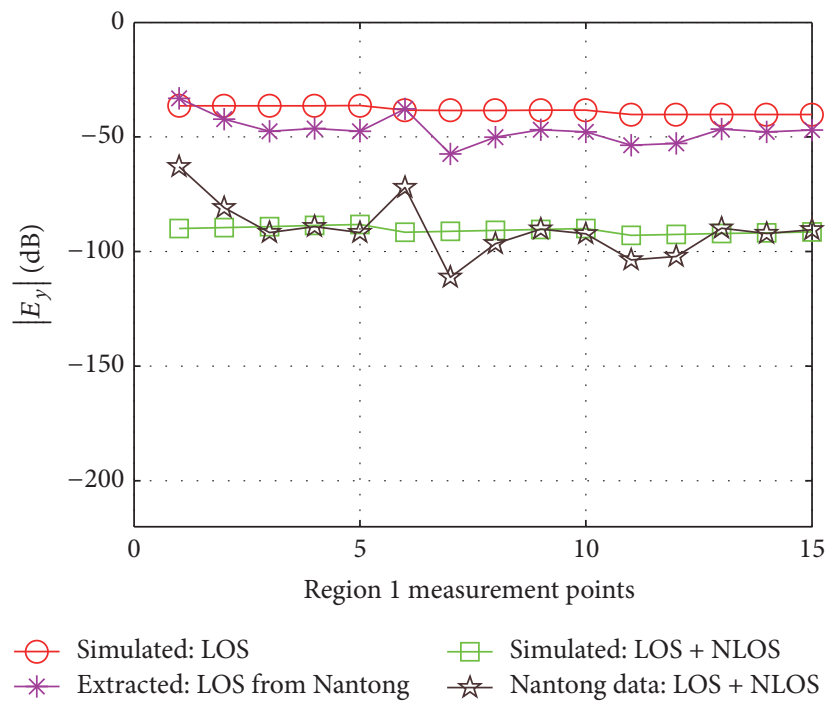

(a)

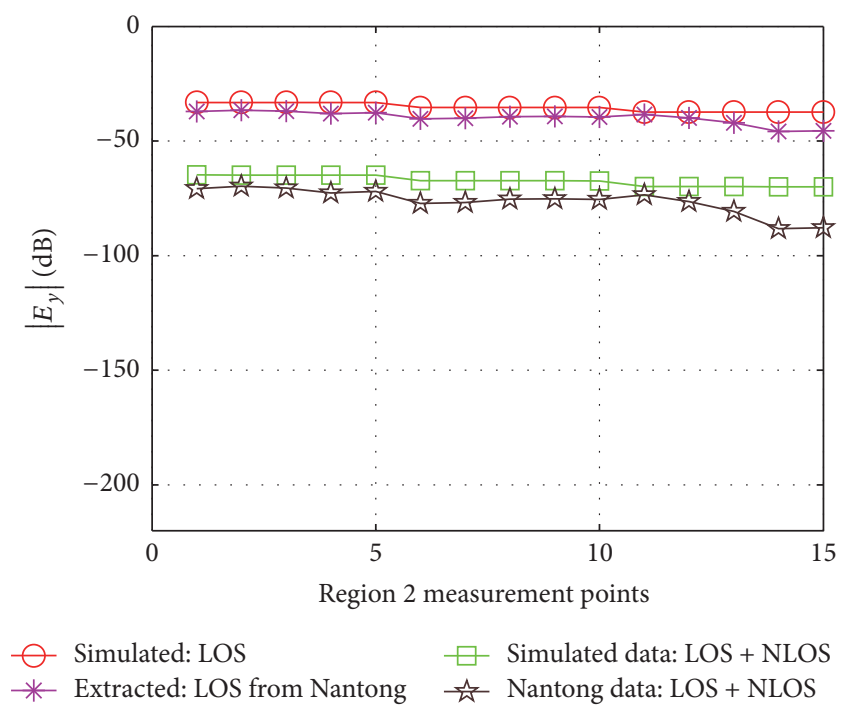

(b)

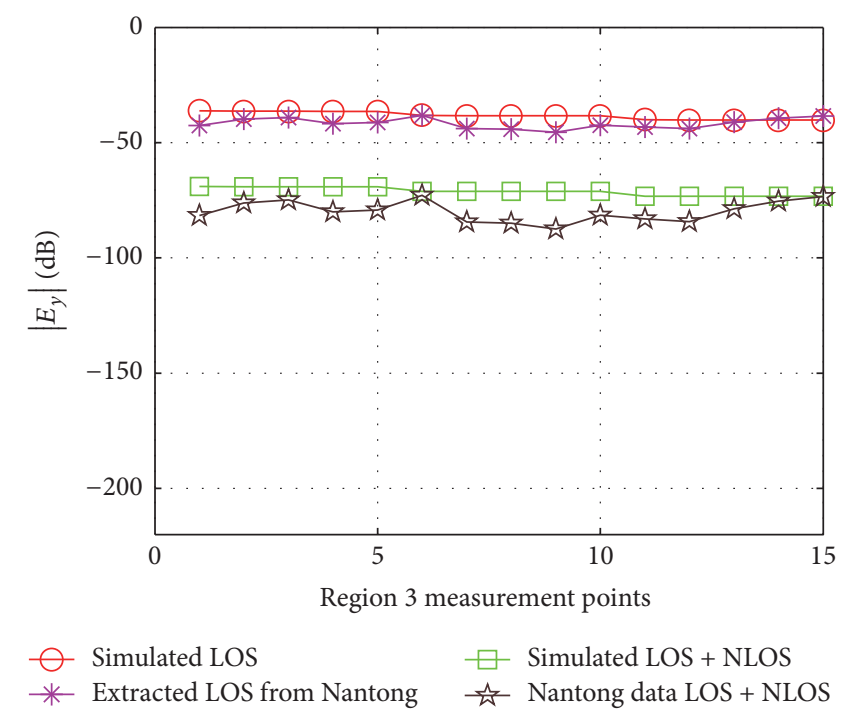

(c)

FIGURE 6: The distribution of $E_{y}$ of horizontally polarized LCX for the LOS and NLOS paths and its comparison with the Nantong Tunnel different measuring locations such as in (a) Region 1, (b) Region 2, and (c) Region 3, respectively.

radiated field. The measured results also show that horizontal polarized LCXs show better performance in terms of radiated field, than the vertically polarized LCXs.

\section{Conclusions}

In this paper, the study on the distribution of the radiated field of LCX for both the LOS and NLOS paths in a confined area by using Ray-Tracing Method as well as radiated theory of slots at $1.8 \mathrm{GHz}$ was carried out. In summary this study concludes as follows:

(i) Minimizing the period of slots in LCX yields a better radiated field performance. (ii) The selection of the appropriate period of slots in LCX and its orientation with respect to the receiving antenna reduces the fluctuation of the electric field.

(iii) The horizontally polarized LCX provides a better performance as compared to vertically polarized LCX.

(iv) In particular, this study reveals a better radiated field in Region 2 in comparison to Region 1 and Region 3.

The reported study is useful to devise an optimum system design. Thus this paper conveys a potential direction for the LTE system design based on the LCX deployment in tunnel. 


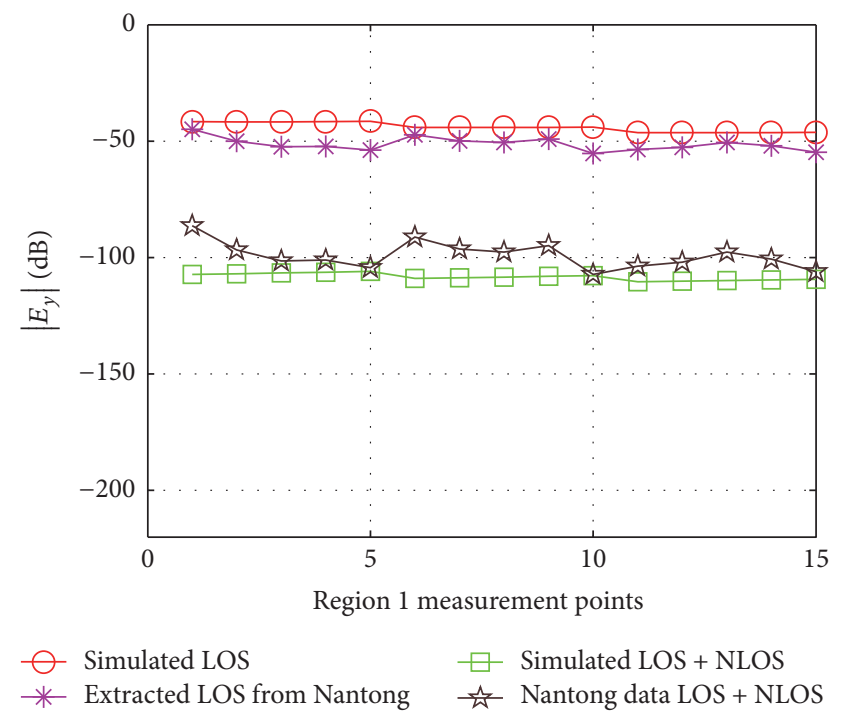

(a)

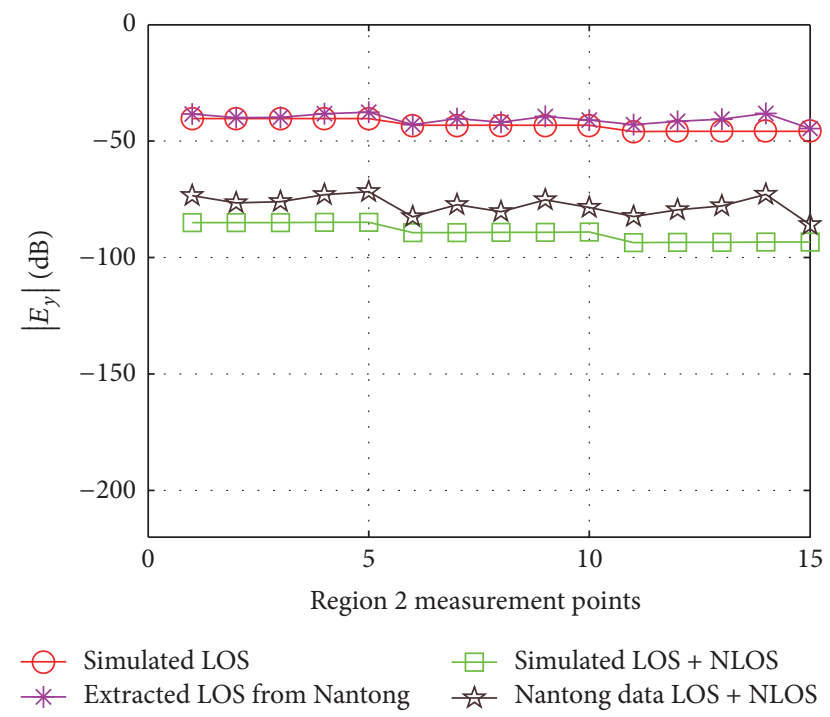

(b)

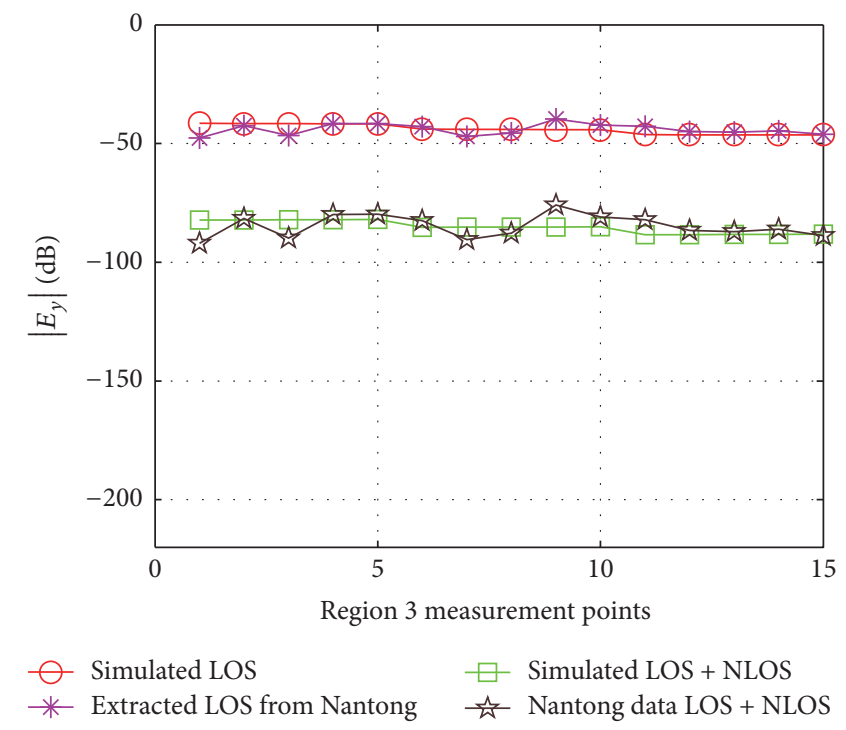

(c)

Figure 7: The distribution of $E_{y}$ of vertically polarized LCX for the LOS and NLOS paths and its comparison with the Nantong Tunnel different measuring locations such as in (a) Region 1, (b) Region 2, and (c) Region 3, respectively.

\section{Conflicts of Interest}

The authors declare that they have no conflicts of interest.

\section{Acknowledgments}

This work was supported by the National Natural Science Foundation of China under Grant 61571282 and the Open Project Program of the State Key Laboratory of Rail Traffic Control and Safety under Grant RCS2017K012. The authors particularly appreciate Mr. Yiming Wu's help during experiments.

\section{References}

[1] S. Banerjee and H. Sharif, "A survey of wireless communication technologies \& their performance for high speed railways," Journal of Transportation Technologies, vol. 6, no. 1, pp. 15-29, 2016.

[2] K.-S. Chung and C. Keum, "Design of dynamic SNS on the high-speed railway: Virtual crew service," in Proceedings of the 2016 International Conference on Information and Communication Technology Convergence, ICTC 2016, pp. 411-413, kor, October 2016.

[3] H. Wang, F. R. Yu, and H. Jiang, "Modeling of radio channels with leaky coaxial cable for LTE-M based CBTC systems," 
IEEE Communications Letters, vol. 20, no. 5, pp. 1038-1041, 2016.

[4] Y. Wu, G. Zheng, A. Saleem, and Y. P. Zhang, "An experimental study of MIMO performance using leaky coaxial cables in a tunnel," IEEE Antennas and Wireless Propagation Letters, vol. 16, pp. 1663-1666, 2017.

[5] J. Wang, Y. Li, Z. Zhang, and M. Chen, "Radiation mechanism and polarization properties of leaky coaxial cables," Radio Science, vol. 46, no. 2, Article ID RS2009, pp. 1-12, 2011.

[6] F. Suzuki, "Thin leaky coaxial cable LCX-5D for $2.4 \mathrm{GHz}$ wireless Lan," Fujikura Technical Review, 2013.

[7] J. H. Wang, "Research on the radiation characteristics of patched leaky coaxial cable by FDTD method and mode expansion method," IEEE Transactions on Vehicular Technology, vol. 57, no. 1, pp. 90-96, 2008.

[8] C. Zhang, J. Wang, M. Chen, and Z. Zhang, "Radiation characteristic of the leaky circular waveguide with periodic slots," IEEE Antennas and Wireless Propagation Letters, vol. 11, pp. 503-506, 2012.

[9] Z. Zixin, Y. Xiaodong, and G. Shuxiang, "A coupling loss algorithm of leaky coaxial cable in the blind zone," in Proceedings of the 2008 IEEE International Conference on Mechatronics and Automation, ICMA 2008, pp. 919-923, Japan, August 2008.

[10] D. H. Kim and H. J. Eom, "Radiation of a leaky coaxial cable with narrow transverse slots," IEEE Transactions on Antennas and Propagation, vol. 55, no. 1, pp. 107-110, 2007.

[11] Y. Wu, G. Zheng, and T. Wang, "Performance analysis of MIMO transmission scheme using single leaky coaxial cable," IEEE Antennas and Wireless Propagation Letters, vol. 16, pp. 298-301, 2017.

[12] F. Fuschini, E. M. Vitucci, M. Barbiroli, G. Falciasecca, and V. Degli-Esposti, "Ray tracing propagation modeling for future small-cell and indoor applications: a review of current techniques," Radio Science, vol. 50, no. 6, pp. 469-485, 2015.

[13] S. P. Morgan, "Prediction of indoor wireless coverage by leaky coaxial cable using ray tracing," IEEE Transactions on Vehicular Technology, vol. 48, no. 6, pp. 2005-2014, 1999.

[14] S. Pu and J. H. Hao, "Integrative modeling of wireless RF links for train-to-wayside communication in railway tunnel," Journal of the Korea Industrial Information Systems Research, vol. 17, no. 2, pp. 19-27, 2012.

[15] X. Zhang, Z. Zhao, X. Yang, L. Guo, and W. Li, "Research on the radiation characteristics of leaky coaxial cable and spiral antenna in the blind zone," in Proceedings of the 2006 International Conference on Communications, Circuits and Systems, ICCCAS, pp. 811-815, china, June 2006.

[16] K. Inomata, W. Tsujita, and T. Hirai, “Two-frequency surveillance technique for intrusion-detection sensor with Leaky Coaxial Cables," in Proceedings of the 2014 9th IEEE Sensors Applications Symposium, SAS 2014, pp. 103-106, New Zealand, February 2014.

[17] L. Feng, Z. Wang, X. Yang, and B. Xu, "Research on the radiation field of wide-band leaky coaxial cable," in Proceedings of the 2010 6th International Conference on Wireless Communications, Networking and Mobile Computing, WiCOM 2010, pp. 1-4, china, September 2010.

[18] J. Wang, "Leaky coaxial cable with circular polarization property," IEEE Transactions on Antennas and Propagation, vol. 59, no. 2, pp. 682-685, 2011.

[19] X. Zhang, X. Yang, L. Guo, and Z. Zhao, "Research on the radiated field's distribution of leaky coaxial cable in the blind zone," in Proceedings of the 2006 1st International Symposium on Systems and Control in Aerospace and Astronautics, pp. 4-7, Harbin, China, 2006.

[20] L. J. Greenstein, D. G. Michelson, and V. Erceg, "Momentmethod estimation of the Ricean $K$-factor," IEEE Communications Letters, vol. 3, no. 6, pp. 175-176, 1999. 


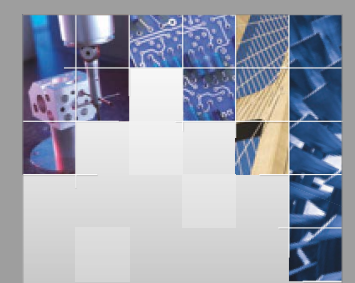

\section{Enfincering}
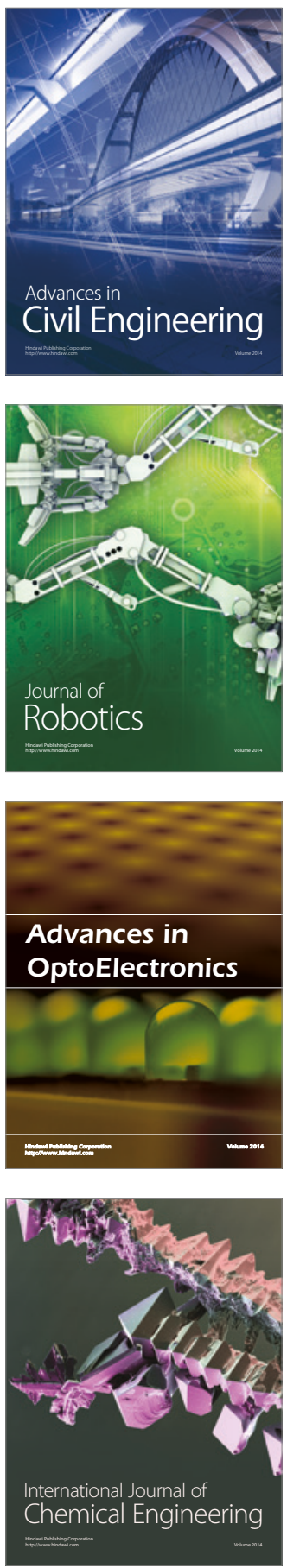

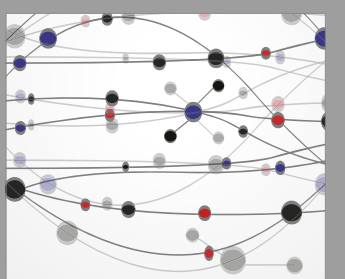

The Scientific World Journal

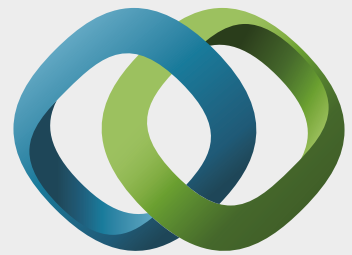

\section{Hindawi}

Submit your manuscripts at

https://www.hindawi.com
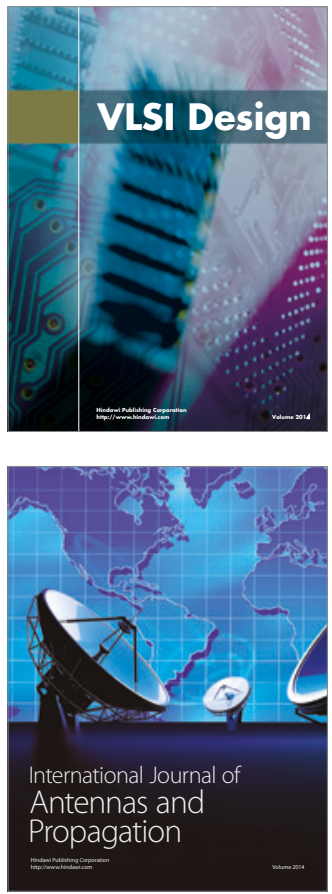

\section{Rotating}

Machinery
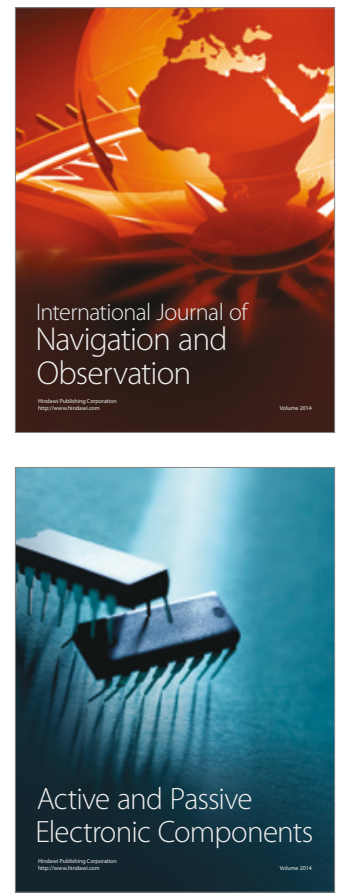
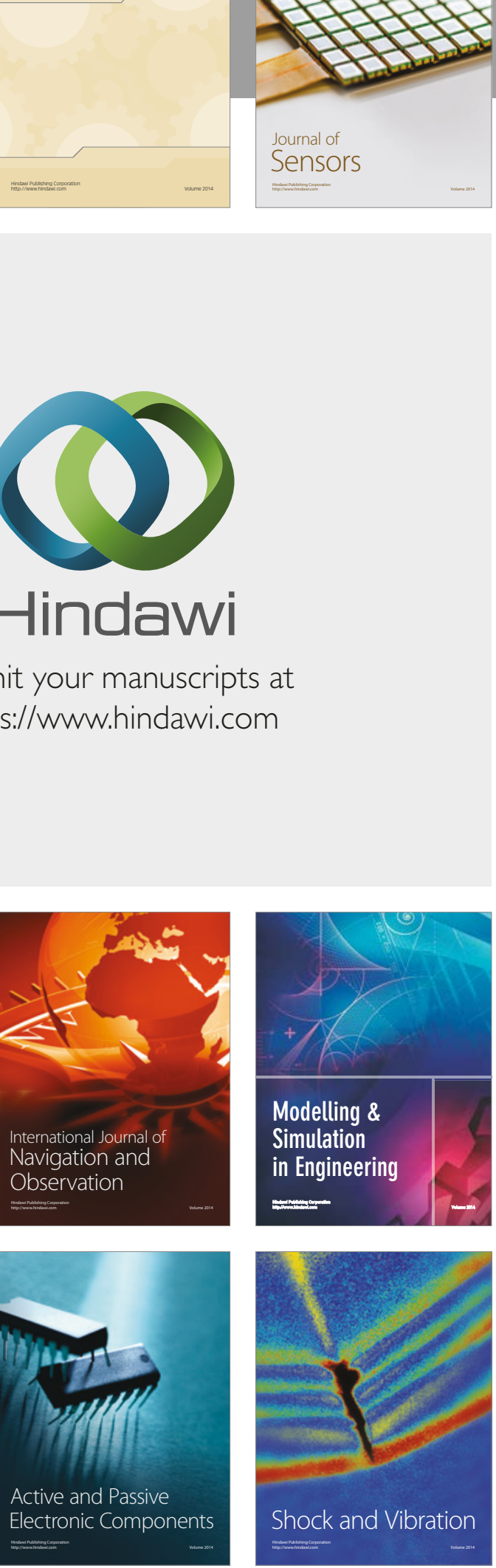
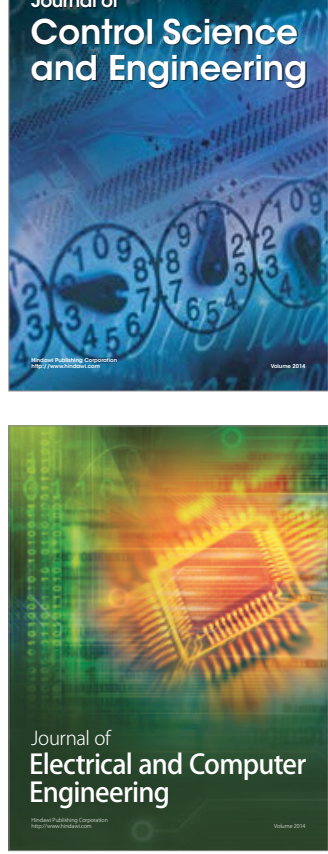

Distributed

Journal of

Control Science

and Engineering
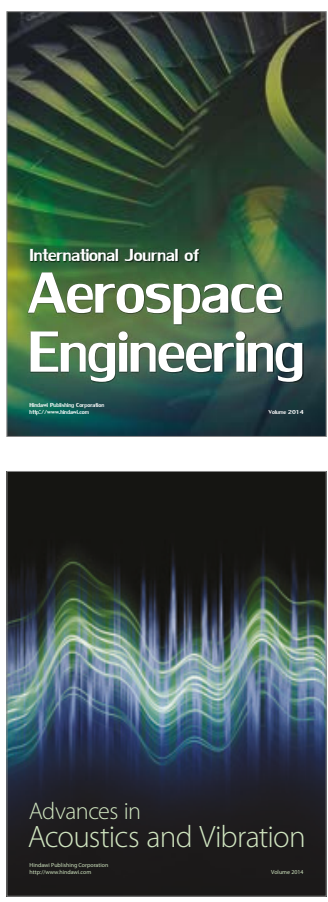

Sensor Networks 